\title{
Effect of Treadmill Training with Ankle Joint Taping on Gait Function and Balance Ability in Patients with Subacute Stroke: A Randomized, Controlled, Preliminary Trial
}

\author{
Yeong-Jin Gill, PT, MS · Se-Jung Oh, PT, MS · Yong-Jun Cha, PT, $\mathrm{PhD}^{1 \dagger}$
}

Dept. of Physical Therapy, Graduate School, Daejeon University

'Dept. of Physical Therapy, College of Health and Medical Science, Daejeon University

Received: November 32021 / Revised: November 32021 / Accepted: December 122021

(c) 2022 J Korean Soc Phys Med

\begin{abstract}
| Abstract |
PURPOSE: This study examined the effects of treadmill training with taping on the affected ankle joint on the gait and balancing ability in patients with sub-acute stroke.

METHODS: Nineteen patients with sub-acute hemiplegic stroke in a rehabilitation hospital were assigned randomly to either the experimental group $(\mathrm{n}=10)$, who received treadmill training with taping on the affected ankle joint or the control group $(\mathrm{n}=9)$, who received general treadmill training. All participants performed $60 \mathrm{~min}$ of comprehensive rehabilitation therapy (five sessions per week for four weeks). Each group received treadmill training with or without taping on the affected ankle joint for $20 \mathrm{~min}$ (three sessions per week for four weeks). The gait and balancing ability were measured before and after the four-week training.
\end{abstract}

RESULTS: Post-training scores of 10 -meter walk test (10 MWT), timed up and go (TUG) test, and center of pressure (COP) path length and velocity for the experimental group

$\dagger$ Corresponding Author : Yong-Jun Cha cha0874@dju.kr, https://orcid.org/0000-0002-8553-7098

This is an Open Access article distributed under the terms of the Creative Commons Attribution Non-Commercial License (http://creativecommons.org/licenses/by-nc/3.0) which permits unrestricted non-commercial use, distribution, and reproduction in any medium, provided the original work is properly cited. increased significantly compared to that pre-training $(\mathrm{p}<.05)$. The experimental group showed a larger decrease in the 10 MWT and TUG test than the control group ( $-3.5 \mathrm{~s}$ vs. -1.01 $\mathrm{s}, \mathrm{p}<.05 ;-4.9$ s vs. $-1.7 \mathrm{~s}, \mathrm{p}<.05$; respectively)

CONCLUSION: Treadmill training with taping on the affected ankle joint might improve the gait and balancing ability of stroke patients and is considered a more effective method for improving gait and balancing ability than the method of general treadmill training.

Key Words: Ankle joint, Balance, Stroke, Taping

\section{Introduction}

Stroke is a disease that causes functional impairment in the body due to a sudden interruption of blood supply to the blood vessels in the brain [1]. The main physical impairments caused by stroke show a reduced walking function and reduced balance ability [2]. When maintaining walking and standing postures, stroke patients develop a weight-bearing imbalance that supports $61-80 \%$ of their total body weight on the non-paralyzed side [3]. To compensate for this, stroke patients move excessively in the lower extremity joint on the non-paraplegic side. More energy may be required for walking than a normal person 
$[4,5]$.

The ankle joint is a major joint that provides weightbearing function and proprioceptive feedback for postural control during standing and walking [6,7]. Paralysis caused by stroke mainly deforms the ankle joint on the paralyzed side into plantar flexion and inversion [8,9]. Deformation of the ankle joint on the paralyzed side is a major factor in increasing the weight bearing on the non-affected side and decreasing the walking speed [10,11]. Various intervention methods such as passive range of motion exercise, active assistance exercise, resistance exercise, ankle joint orthosis, and taping have been performed to minimize or correct the deformity of the ankle joint on the paralyzed side [12-15]. Ankle joint taping was effective in activating the tibialis anterior and calf muscles on the paralyzed side of stroke patients [16], and it is reported to be effective in improving the balance and walking ability by promoting proprioception in the ankle joint [17]. In addition, taping applied to the ankle joint was reported to be effective in stabilizing the stance phase of the paralyzed foot [18].

Treadmill training is a major intervention method generally used to improve the gait function in patients with subacute or chronic stroke [19]. Treadmill training effectively improves the gait speed and endurance in stroke patients [20]. This training was reported to effectively reduce weight-bearing imbalances by increasing the stance period of the paralyzed lower extremity and reducing the increased energy consumption caused by abnormal gait patterns [21].

Most of these studies that investigated the effect of taping on stroke patients have investigated the effect of taping while walking on the ground or in a static standing posture $[22,23]$. Although treadmill training in previous studies was as effective in improving the gait and balance ability of stroke patients as the ground gait training $[19,20]$, studies on the effect of taping applied directly during treadmill training are insufficient. Therefore, this study examined the effect of taping applied during treadmill training, which is generally performed to improve the walking function and balance ability of stroke patients.

This study hypothesized that treadmill training with taping applied to the paralyzed ankle joint would be more effective in improving the gait function and balance ability of subacute stroke patients than treadmill training conducted without taping.

\section{Methods}

\section{Participants}

This preliminary experimental study was conducted on subacute patients with an onset period of fewer than six months hospitalized at Da Vinci Hospital in Daejeon Metropolitan City. The inclusion criteria were as follows. Those with less than six months of stroke onset, a score of 24 or higher on the MMSE-K (Korean version of mini-mental status examination)[24], those who can walk more than $10 \mathrm{~m}$ independently with or without a walking aid, those who understand the purpose of the study and provided written consent form. The exclusion criteria were as follows. Those with a history of surgery on the lower extremities, high skin sensitivity or skin diseases, those who complained of pain during dorsiflexion of the ankle joint, and those with a modified Ashworth Scale $>2$.

Of the 45 subacute stroke patients, 19 were finally selected according to the selection and exclusion criteria. The purpose and procedure of this study were explained to the subjects verbally. All 19 subjects consented voluntarily to participate in the experiment. This study was conducted under the approval of the Research Ethics Committee of Daejeon University (IRB No. 1040647202104-HR-004-03).

Nineteen subjects were assigned randomly into two groups through a lottery process: 10 subjects (male 5 and female 5; mean age $57.00 \pm 7.67$ years) in the experimental group who performed treadmill training with taping applied 
to the ankle joint and 9 subjects (male 5 and female 4; mean age $58.55 \pm 17.89$ years) in the control group who received treadmill training without applying taping.

\section{Intervention}

A treadmill (Fitex-5080, Fitex Ind., Gyeonggi-do, Korea) with easy speed control was used for gait training. All subjects underwent comprehensive rehabilitation, which included the joint range of motion exercise, muscle strength exercise, balance training, and gait training for 60 minutes, five times a week, for a total of four weeks. In addition, treadmill walking training with ankle taping or treadmill walking without ankle taping was performed for 20 minutes, three times a week, for four weeks.

1) Experimental group (aping treadmill-training group) For taping treadmill training, kinesio tape (Benefact tape, Sigmax, Japan) was used for ankle taping. Kinesio tape is used widely to stabilize the ankle joint during static standing or walking in stroke patients [25]. In this study, the tape was attached to the peroneus tertius, peroneus longus, tibialis anterior, and extensor hallucis longus, which are used widely for ankle joint stability in stroke patients $[26,22]$. One physical therapist with three years of clinical experience applied the tape at the position where the muscle was maximally elongated. To attach the tape to the peroneus tertius, one end of the tape was fixed to the 5th metatarsal on the paralyzed side with the ankle joint supinated, and was attached to the fibular $1 / 3$ area along the direction of the muscle fiber running. To attach the tape to the peroneus longus, one end was fixed to the base of the first metatarsal with the ankle joint supinated, and was attached to the lateral process of fibular along the direction of the muscle fiber running. To attach the tape to the tibialis anterior, one end was fixed between the big toe and the index toe on the paralyzed side with the ankle joint pronated and was attached to the outside of the knee along the direction of the muscle fiber running. To attach the tape to the extensor digitorum longus, one end of the tape was

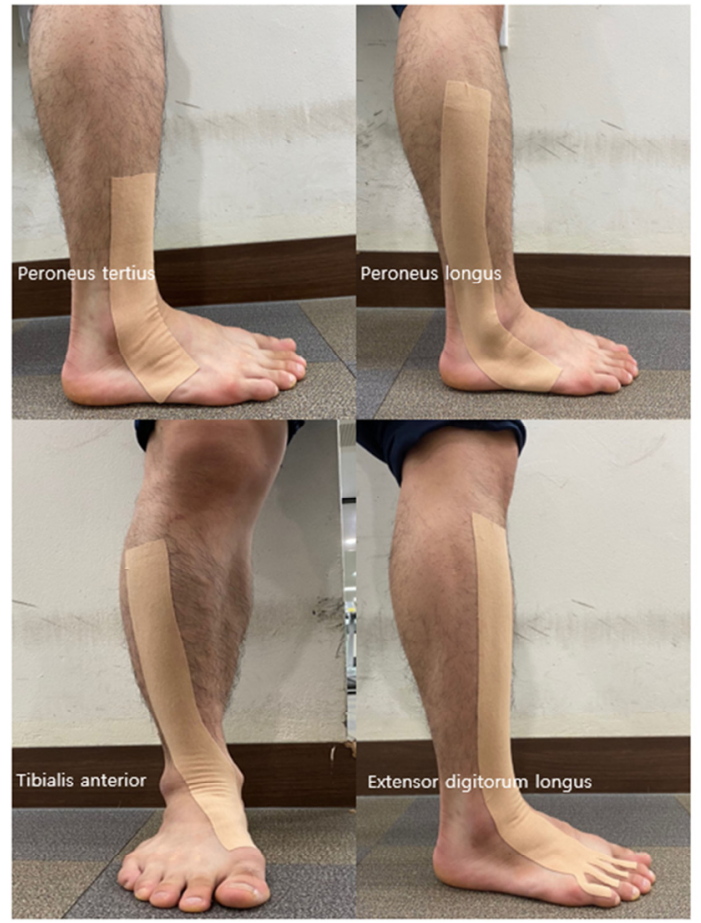

Fig. 1. Taping position applied to the ankle joint on the paralyzed side of a stroke patient.

divided into four parts, and the tape was applied from the second to fifth toe on the paralyzed side to the fibular 2/3 area (Fig. 1). With kinesio tape attached to the four muscles, each participant was trained to walk on a treadmill for 20 minutes. A physical therapist with three years of clinical experience blinded to the purpose of this study applied treadmill training to each subject. Treadmill walking speed was applied by checking the condition of each participant on the day before the intervention. Treadmill training speed was applied at the level of Borg scale level 10 to 12 (comfortable speed) [27]. The training was immediately stopped if the subject complained of fatigue during treadmill training; training was resumed after sufficient rest.

2) Control group (general treadmill training group) General treadmill training without taping was carried out for the same time as the gait training of the experimental 
group, and the treadmill speed was also performed at a comfortable speed in the same manner as in the experimental group.

\section{Outcome Measures}

In this study, the gait function and balance ability were measured to compare the effects of treadmill training in stroke patients. All measurements were taken by a physical therapist with three years of clinical experience who did not participate in the randomization of subjects in this study. Gait function was measured by the walking speed and walking endurance. The balance ability was measured by dynamic and static balance ability. In both groups, the gait function and balance ability were measured before treadmill training and after four weeks of treadmill training. Each measurement variable was measured three times to calculate the average value.

\section{1) Walking speed test}

A $10 \mathrm{~m}$ walking speed test (10 MWT) was performed to evaluate the walking speed. 10 MWT is a useful method for evaluating the walking speed of stroke patients, and has high reliability with inter- and intra-rater reliability $\mathrm{r}=.95 \sim .96[28,29]$. In this study, the average time was finally recorded by measuring three times for each participant.

2) Walking endurance test

A three-minute gait test (3 MWT) was performed to test the gait endurance. The three-minute gait test is a test to measure gait endurance. In a straight-line distance of $30 \mathrm{~m}$, the participant was asked to walk back and forth as fast as possible for three minutes. It is a method of measuring the number of round trips and the total distance traveled. The 3 MWT showed high intra-measurement reliability (ICC $=.85$ to .97 ) in measuring the walking endurance in chronic stroke patients [30]. The examiner informed the participant of the passage of time every minute to reinforce the participant's gait concentration.

3) Dynamic balancing ability test

The timed up and go (TUG) test was performed to test the dynamic balance ability. The TUG test is a method of measuring the movement ability, which indicates dynamic balance ability. This is a method of measuring the time taken to sit on a chair $50 \mathrm{~cm}$ high and return to the chair by going around a turning point at a distance of $3 \mathrm{~m}$. This test method showed high intra- and inter-rater reliability [31]. In this study, the average time was recorded after measuring three times.

\section{4) Static balancing ability test}

The static balance ability was measured using the Balancia 2.0 program (Mintosys, Seoul, Korea). The Balancia program collects information on the center of pressure (COP) while a patient maintains a static standing balance on the Nintendo Wii Balance Board (Nintendo, Tokyo, Japan). The Wii Balance Board is 20.5 in. $\times 13.2$ in. $\times 3.1$ in. in size with a rectangular shape, and the COP information is collected simultaneously through load cells located at each corner and saved to a computer via a Bluetooth connection. The path length and velocity (Vel avg.) for $\mathrm{X}$ and $\mathrm{Y}$ of $\mathrm{COP}$ were analyzed in this study. The test-retest reliability (ICC) of COP measurement through the Balancia program is .66 .94, indicating high reliability [32]. To evaluate the static balance ability, all patients stood placed on the Wii Balance Board with their feet shoulder-width apart, arms stretched out comfortably, head facing forward, and eyes opened for $30 \mathrm{~s}$. Investigators who did not participate in the screening process of this study evaluated the static balance ability and calculated the average after three repeated measurements.

\section{Data Treatment}

All collected data were analyzed using the SPSS version 25.0 statistical program (IBM Corp., Armonk, NY, USA) 


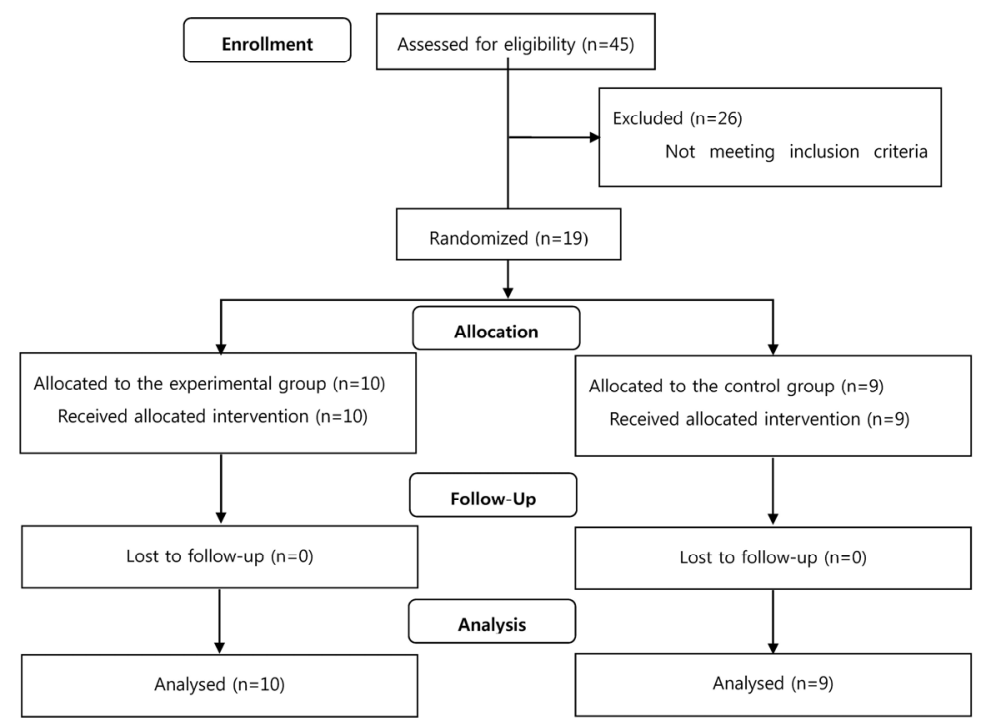

Fig. 2. CONSORT diagram showing study flow.

Table 1. Demographic Characteristics of the Participants at the Baseline

\begin{tabular}{lcccc}
\hline \multicolumn{1}{c}{ Demographic Features } & Experimental Group $(\mathrm{n}=10)$ & Control Group $(\mathrm{n}=9)$ & $x^{2} / t$ & $P$ \\
\hline Gender (male/female) & $5 / 5$ & $5 / 4$ & .059 & 1.000 \\
Age (years) & $57.00(7.67)$ & $58.55(17.89)$ & .251 & .805 \\
Height (cm) & $165.10(7.78)$ & $166.55(9.90)$ & .358 & .725 \\
Weight (kg) & $65.10(9.59)$ & $66.97(11.36)$ & .389 & .702 \\
Paretic side (right/left) & $6 / 4$ & $4 / 5$ & .460 & .656 \\
Onset duration (days) & $84.80(31.77)$ & $110.33(56.89)$ & 1.225 & .237 \\
Stroke type (infarction/hemorrhage) & $4 / 6$ & $6 / 3$ & 1.351 & .370 \\
MMSE-K & $27.50(1.50)$ & $28.33(1.50)$ & 1.205 & .245 \\
\hline
\end{tabular}

Values are expressed frequency or mean (SD).

Experimental group, treadmill training with taping on the affected ankle joint; Control group, general treadmill training MMSE-K, Korean version of the mini-mental status examination.

for Windows. An independent sample t-test and chi-square test were performed to determine the group homogeneity between the experimental and control groups. A Wilcoxon signed-rank test was performed to compare the gait function and balance ability before and after intervention for four weeks in each group. The Mann-Whitney U-test was performed to compare the change before and after the intervention between the two groups. The statistical significance level was set at .05

\section{Results}

Of the 20 subjects, one control patient was discharged from the hospital during the intervention period. The participants who completed the four-week intervention were 10 in the experimental group and nine in the control group (Fig. 2). Table 1 lists the general characteristics of the 19 subjects, who finally completed the study. There were no significant differences between the two groups 
Table 2. Pre- to Post-training Changes in Balance Variables in the Two Study Groups

\begin{tabular}{|c|c|c|c|c|}
\hline & & Experimental Group $(\mathrm{n}=10)$ & Control Group $(n=9)$ & $Z$ \\
\hline \multirow{4}{*}{10 MWT (m/sec) } & Pre & $15.56 \pm 8.14$ & $17.28 \pm 9.61$ & -.245 \\
\hline & Post & $12.04 \pm 6.19$ & $16.27 \pm 8.66$ & -1.388 \\
\hline & $Z$ & $-2.803^{\mathrm{a}}$ & -.059 & \\
\hline & $\triangle($ post-pre $)$ & $-3.52 \pm 3.07$ & $-1.01 \pm 4.65$ & $-2.123 b$ \\
\hline \multirow{4}{*}{3 MWD (m) } & Pre & $122.49 \pm 50.02$ & $123.26 \pm 72.53$ & -.163 \\
\hline & Post & $135.33 \pm 64.44$ & $128.17 \pm 68.05$ & -.408 \\
\hline & $Z$ & -1.580 & -.533 & \\
\hline & $\triangle($ post-pre $)$ & $12.83 \pm 29.81$ & $4.92 \pm 18.12$ & -.980 \\
\hline \multirow{4}{*}{ TUG $(\mathrm{m} / \mathrm{sec})$} & Pre & $17.34 \pm 8.69$ & $19.81 \pm 9.30$ & -.816 \\
\hline & Post & $12.47 \pm 5.94$ & $18.08 \pm 8.63$ & -1.470 \\
\hline & $Z$ & $-2.803^{\mathrm{a}}$ & -.533 & \\
\hline & $\triangle($ post-pre $)$ & $-4.86 \pm 4.44$ & $-1.73 \pm 4.98$ & $-1.715^{\mathrm{b}}$ \\
\hline \multirow{4}{*}{$\begin{array}{l}\text { COP path length } \\
(\mathrm{mm})\end{array}$} & Pre & $86.15 \pm 23.47$ & $90.31 \pm 26.83$ & -.327 \\
\hline & Post & $75.62 \pm 14.19$ & $81.49 \pm 23.07$ & -.082 \\
\hline & $Z$ & $-2.191^{\mathrm{a}}$ & $-1.955^{\mathrm{a}}$ & \\
\hline & $\triangle($ post-pre $)$ & $-10.53 \pm 13.94$ & $-8.82 \pm 11.54$ & -.408 \\
\hline \multirow{4}{*}{$\begin{array}{l}\text { COP path velocity } \\
(\mathrm{cm} / \mathrm{sec})\end{array}$} & Pre & $2.87 \pm .78$ & $2.97 \pm .85$ & -.245 \\
\hline & Post & $2.52 \pm .47$ & $2.72 \pm .77$ & -.082 \\
\hline & $Z$ & $-2.191^{\mathrm{a}}$ & -.889 & \\
\hline & $\triangle$ (post-pre) & $-.35 \pm .47$ & $-.25 \pm .42$ & -.735 \\
\hline
\end{tabular}

Values are expressed as the mean $( \pm \mathrm{SD})$

Experimental group, treadmill training with taping on the affected ankle joint; Control group, general treadmill training 3 MWD, 3-minute walking distance; TUG, timed up and go test; COP, center of pressure.

${ }^{a}$ Significantly different $(p<.05)$ from the pre-tests

${ }^{\mathrm{b}}$ Significantly different $(p<.05)$ in gains between the two groups

regarding gender, paralysis side, cause of disease, age, onset period, height, weight, and MMSE-K ( $p>.05)$.

Table 2 lists the changes in gait function and balance ability before and after the training of the two groups. In the experimental group, there was a significant improvement in the 10 MWT, TUG test, COP path length, and COP path velocity after training compared to that before training $(\mathrm{p}<.05)$. In 10 MWT, it decreased by $3.5(\mathrm{~m} / \mathrm{s})$ compared to that before the intervention, and it decreased by $4.9 \mathrm{~s}$ in TUG. The COP path length decreased by .35 $\mathrm{cm}$, and the COP path velocity decreased by $10.5 \mathrm{~cm} / \mathrm{s}$. In the control group, there was a significant decrease in the COP path velocity after training compared to that before the intervention $(p<.05)$. A comparison of changes in gait function and balance ability before and after training in the experimental group and the control group revealed a significant difference between the $10 \mathrm{MWT}$ and TUG test $(\mathrm{p}<.05)$. The experimental group decreased more than the control group: by $2.5 \mathrm{~s}$ in 10 MWT and $3.1 \mathrm{~s}$ in the TUG test. 


\section{Discussion}

This study compared the intervention effect of treadmill training with ankle joint kinesio taping and treadmill training without taping for adult patients with subacute stroke. Treadmill training with taping showed significant improvement after intervention in gait speed and static and dynamic balance ability except for gait endurance. Furthermore, there was a more significant improvement in the walking speed and dynamic balance ability than in the control group. These results are partially consistent with the hypothesis of this study, which showed that the treadmill training applied with taping is more effective than general gait training.

Jackson applied kinesio taping to the ankle joint for stroke patients and performed static standing training on a balance pad. As a result, kinesio taping improved the balance ability by $24 \%$ compared to before training [33]. Choi et al. reported that when kinesio taping was applied to the ankle joint on the paralyzed side of stroke patients, the TUG test time decreased by 2.8 seconds and by .2 seconds at 10 MWT $[22,34]$. These results are similar to the results in the present study in which ankle joint kinesio taping treadmill training in this study reduced 10 MWT and TUG tests significantly. Kim et al. reported that kinesio taping applied to the ankle joint on the paretic side of stroke patients increased the walking speed of stroke patients [18]. These results support the results of this study in that treadmill training with taping increased the walking speed and dynamic balance ability more than general treadmill training. Kim et al. reported that kinesio taping used to immobilize the joints of stroke patients was effective in muscle activation by stimulating the skin receptors and gamma motor neuron sensitivity in the joint area [18]. Therefore, kinesio taping applied to the ankle joint in this study ultimately contributed to joint stabilization by increasing the muscle activity in the paralyzed ankle joint region.
The COP pathway distance and COP pathway velocity during static standing posture maintenance are major variables in estimating the effect of interventions on balance ability in stroke patients [23]. In this study, the COP pathway distance and COP pathway velocity were decreased significantly in treadmill training with taping. This means that treadmill training with taping is effective in improving the static balance ability of stroke patients. This result is because taping provided an environment in which contraction of the tibialis anterior and peroneus muscles occurred easily. The contraction of both muscles would have had a positive effect on the normalization of COP from heel strike to toe-off of the paralyzed foot [35]. This effect is ultimately considered to have contributed to COP stabilization when maintaining a static standing posture [23]. Abian-Vicen et al. reported that kinesio taping applied to the ankle joint while maintaining one-legged standing in normal adults reduced the left-right movement distance of the COP pathway by $3.2 \mathrm{~cm}$ more than when no taping was applied [36]. Kwak reported that kinesio taping applied to the tibialis anterior decreased the COP pathway distance by $1.18 \mathrm{~cm}$ and the COP pathway velocity by $.03 \mathrm{~cm} / \mathrm{s}$ when maintaining a static standing posture in stroke patients [37]. These results support the current results because treadmill training with taping reduced the COP pathway distance and velocity of stroke patients.

This study had some limitations. First, this study is a pilot study investigating the effects of kinesio taping on gait function and balance ability during treadmill training in stroke patients. Owing to the small number of study subjects, the main intervention method of this study cannot be generalized and applied. Second, this study was conducted on subacute patients admitted to a rehabilitation hospital. Considering the length of stay, this study had no choice but to apply the four-week intervention period. Hence, there are limitations in determining the long-term effects of interventions. These limitations make it difficult to generalize the results and apply them to treadmill training 
for stroke patients. On the other hand, this study will be clinically meaningful in that it is the first study to examine the effects of ankle joint taping during treadmill training, which is generally performed to improve the walking function and balance ability of stroke patients. In the future, based on these results, it is expected that the most effective taping method for improving the function of stroke patients can be suggested by comparing the intervention effect according to the taping type.

\section{Conclusion}

This study examined the effect of taping on the paralyzed ankle joint during treadmill training on the gait function and balance ability in subacute stroke patients. As a result, treadmill training with taping improved the gait function and balance ability of stroke patients. Taping was more effective in improving the gait speed and dynamic balance than general treadmill training. Therefore, taping the ankle joint of the paralyzed side during treadmill training for stroke patients is expected to be an effective intervention for improving the gait and balance ability of stroke patients.

\section{References}

[1] Haruyama K, Kawakami M, Otsuka T. Effect of Core Stability Training on Trunk Function, Standing Balance, and Mobility in Stroke Patients. Neurorehabil Neural Repair. 2017;31(3):240-9.

[2] Yang YR, Tsai MP, Chuang TY, et al. Virtual reality-based training improves community ambulation in individuals with stroke: a randomized controlled trial. Gait Posture. 2008;28(2):201-6.

[3] Csapo R, Alegre LM. Effects of Kinesio( $(\mathbb{B})$ taping on skeletal muscle strength-A meta-analysis of current evidence. J Sci Med Sport. 2015;18(4):450-6.

[4] Granat MH, Maxwell DJ, Ferguson AC, et al. Peroneal stimulator; evaluation for the correction of spastic drop foot in hemiplegia. Arch Phys Med Rehabil. 1996;77(1): 19-24.

[5] Horstman AM, Beltman MJ, Gerrits KH, et al. Intrinsic muscle strength and voluntary activation of both lower limbs and functional performance after stroke. Clin Physiol Funct Imaging. 2008;28(4):251-61.

[6] Runge CF, Shupert CL, Horak FB, et al. Ankle and hip postural strategies defined by joint torques. Gait Posture. 1999;10(2):161-70.

[7] Menz HB, Lord SR, Fitzpatrick RC. Acceleration patterns of the head and pelvis when walking on level and irregular surfaces. Gait Posture. 2003;18(1):35-46.

[8] Laufer Y. The effect of walking aids on balance and weight-bearing patterns of patients with hemiparesis in various stance positions. Phys Ther. 2003;83(2):112-22.

[9] Wong AM, Pei YC, Hong WH, et al. Foot contact pattern analysis in hemiplegic stroke patients: an implication for neurologic status determination. Arch Phys Med Rehabil. 2004;85(10):1625-30.

[10] Chu KS, Eng JJ, Dawson AS, et al. Water-based exercise for cardiovascular fitness in people with chronic stroke: a randomized controlled trial. Arch Phys Med Rehabil. 2004;85(6):870-4.

[11] Kim W. The effects of clinical characteristics of chronic stroke patients on physiological cost index during walking. Korean Research Society of Physical Therapy. 2006;13(1): 32-7.

[12] Guerra Padilla M, Molina Rueda F, Alguacil Diego IM. Effect of ankle-foot orthosis on postural control after stroke: a systematic review. Neurologia. 2014;29(7): 423-32.

[13] Grampurohit N, Pradhan S, Kartin D. Efficacy of adhesive taping as an adjunt to physical rehabilitation to influence outcomes post-stroke: a systematic review. Top Stroke Rehabil. 2015;22(1):72-82.

[14] Wist S, Clivaz J, Sattelmayer M. Muscle strengthening for hemiparesis after stroke: A meta-analysis. Ann Phys Rehabil Med. 2016;59(2):114-24. 
[15] Kim Y, Lai B, Mehta T, et al. Exercise Training Guidelines for Multiple Sclerosis, Stroke, and Parkinson Disease: Rapid Review and Synthesis. Am J Phys Med Rehabil. 2019;98(7):613-21.

[16] Guner S, Alsancak S, Koz M. Effect of two different kinesio taping techniques on knee kinematics and kinetics in young females. J Phys Ther Sci. 2015;27(10):3093-6.

[17] Macgregor K, Gerlach S, Mellor R, et al. Cutaneous stimulation from patella tape causes a differential increase in vasti muscle activity in people with patellofemoral pain. J Orthop Res. 2005;23(2):351-8.

[18] Kim WI, Choi YK, Lee JH, et al. The effect of muscle facilitation using kinesio taping on walking and balance of stroke patients. J Phys Ther Sci. 2014;26(11):1831-4.

[19] Moseley AM, Stark A, Cameron ID, et al. Treadmill training and body weight support for walking after stroke. Stroke. 2003;34(12):3006.

[20] Enam N, Veerubhotla A, Ehrenberg N, et al. Augmented-reality guided treadmill training as a modality to improve functional mobility post-stroke: A proof-ofconcept case series. Top Stroke Rehabil. 2021;28(8): 624-30.

[21] Park SH, Cha YH, Choi YH. Effects of treadmill walking training with randomized walking speed on pulmonary function in persons with chronic stroke. J Korean Soc Phys Med. 2016;11(4):71-8.

[22] Choi SH, Lim CG. Immediate Effects of Ankle Non-elastic Taping on Balance and Gait Ability in Patients With Chronic Stroke: A Randomized, Controlled Trial. J Manipulative Physiol Ther. 2020;43(9):922-9.

[23] Park SJ, Kim TH, Oh S. Immediate Effects of Tibialis Anterior and Calf Muscle Taping on Center of Pressure Excursion in Chronic Stroke Patients: A Cross-Over Study. Int J Environ Res Public Health. 2020;17(11):4109.

[24] Park JE, Lee JH, Cha YJ. The effect of treadmill gait training in an adjusted position from functional training system on chronic stroke patients' walking and balance ability. J Korean Soc Phys Med. 2017;12(1):35-42.
[25] Yazici G, Guclu-Gunduz A, Bayraktar D, et al. Does correcting position and increasing sensorial input of the foot and ankle with Kinesio Taping improve balance in stroke patients? NeuroRehabilitation. 2015;36(3): 345-53.

[26] Choi YK, Nam CW, Lee JH, et al. The Effects of Taping Prior to PNF Treatment on Lower Extremity Proprioception of Hemiplegic Patients. J Phys Ther Sci. 2013;25(9): 1119-22.

[27] Scherr J, Wolfarth B, Christle JW, et al. Associations between Borg's rating of perceived exertion and physiological measures of exercise intensity. Eur J Appl Physiol. 2013;113(1):147-55.

[28] Dean CM, Richards CL, Malouin F. Task-related circuit training improves performance of locomotor tasks in chronic stroke: a randomized, controlled pilot trial. Arch Phys Med Rehabil. 2000;81(4):409-17.

[29] van Loo MA, Moseley AM, Bosman JM, et al. Test-re-test reliability of walking speed, step length and step width measurement after traumatic brain injury: a pilot study. Brain Inj. 2004;18(10):1041-8.

[30] Peters DM, Middleton A, Donley JW, et al. Concurrent validity of walking speed values calculated via the GAITRite electronic walkway and 3 meter walk test in the chronic stroke population. Physiother Theory Pract. 2014;30(3):183-8.

[31] Podsiadlo D, Richardson S. The timed "Up \& Go": a test of basic functional mobility for frail elderly persons. J Am Geriatr Soc. 1991;39(2):142-8.

[32] Park DS, Lee DY, Choi SJ, et al. Reliability and validity of the Balancia using Wii balance board for assessment of balance with stroke patients. Journal of the Korea Academia-Industrial Cooperation Society. 2013;14(6): 2767-72.

[33] Jackson K, Simon JE, Docherty CL. Extended use of Kinesiology Tape and Balance in Participants with Chronic Ankle Instability. J Athl Train. 2016;51(1):16-21.

[34] Choi YS, Kim DW, Jung SM, et al. Effects of paretic 
ankle kinesio-taping on lower leg muscles activity and gait in chronic stroke patients. Neurotherapy. 2021; 25(2):51-9.

[35] Kim NH, Park HY, Son JK, et al. Comparison of underwater gait training and overground gait training for improving the walking and balancing ability of patients with severe hemiplegic stroke: A randomized controlled pilot trial.
Gait Posture. 2020;80:124-9.

[36] Abian-Vicen J, Algre LM, Fernandez-Rodriguez JM, et al. Ankle taping does not impair performance in jump or balance tests. J Sports Sci Med. 2008;7(3):350-6.

[37] Kwak WR. The effects of taping methods applied onto ankle joint on the balance and gait of stroke patients. Master's Degree. Daegu University. 2012. 